\title{
Search for muon to electron neutrino oscillations
}

\author{
The CHARM II Collaboration
}

P. Vilain ${ }^{1)}$, G. Wilquet ${ }^{1)}$

Inter-University Institute for High Energies (ULB-VUB), Brussels, Belgium.

R. Beyer ${ }^{2}$, W. Flegel, T. Mouthuy ${ }^{3)}$, H. Øverås, J. Panman, A. Rozanov ${ }^{4)}$, K. Winter, G. Zacek $^{5)}$, V. Zacek ${ }^{6)}$

CERN, Geneva, Switzerland.

F.W. Büsser, C. Foos, L. Gerland, T. Layda, F. Niebergall, G. Rädel ${ }^{2)}$, P. Stähelin, T. Voss ${ }^{2}$

II. Institut für Experimentalphysik ${ }^{a}$, Universität Hamburg, Hamburg, Germany

D. Favart, Gh. Grégoire, E. Knoops ${ }^{8}$, V. Lemaître Université Catholique de Louvain, Louvain-la-Neuve, Belgium

P. Gorbunov, E. Grigoriev, V. Khovansky, A. Maslennikov Institute for Theoretical and Experimental Physics, Moscow, Russia

W. Lippich, A. Nathaniel ${ }^{9)}$, A. Staude, J. Vogt

Sektion Physik $^{\text {a) }}$ der Universität München, Munich, Germany

A.G. Cocco, A. Ereditato, G. Fiorillo, F. Marchetti-Stasi, V. Palladino, P. Strolin

Università 'Federico II' and INFN Sezione di Napoli, Naples, Italy

A. Capone, D. De Pedis, U. Dore, A. Frenkel-Rambaldi, P.F. Loverre, D. Macina ${ }^{9)}$, G. Piredda, R. Santacesaria

Università 'La Sapienza' and INFN Sezione di Roma, Rome, Italy

E. Di Capua, S. Ricciardi, B. Saitta

Università di Ferrara and INFN Sezione di Ferrara, Ferrara, Italy

B. Akkus, E. Arik, M. Serin-Zeyrek, R. Sever, P. Tolun, M. T. Zeyrek

High Energy Physics Research Centre, YEFAM, Ankara, Turkey

K. Hiller, R. Nahnhauer, H. E. Roloff

DESY- Institut für Hochenergiephysik, Zeuthen, Germany

(Submitted to Zeitschrift für Physik C)

\footnotetext{
Abstract

A search for $\nu_{\mu} \rightarrow \nu_{e}$ and $\bar{\nu}_{\mu} \rightarrow \bar{\nu}_{e}$ oscillations has been carried out with the CHARM II detector exposed to the CERN wide band neutrino beam. The data were collected over five years, alternating beams mainly composed of muon-neutrinos and muon-antineutrinos. The number of interactions of $\nu_{e}$ and $\bar{\nu}_{e}$ observed is comparable with the number of events expected from flux calculations. For large squared mass differences the upper limits obtained on the mixing angle are $\sin ^{2} 2 \theta<9.4 \cdot 10^{-3}$ for $\nu_{\mu}$ oscillating to $\nu_{e}$ and $\sin ^{2} 2 \theta<4.8 \cdot 10^{-3}$ for $\bar{\nu}_{\mu}$ to $\bar{\nu}_{e}$, at the $90 \%$ confidence level. Combining neutrino and antineutrino data the upper limit is $5.6 \cdot 10^{-3}$.

1) National Foundation for Scientific Research, Belgium

2) Now at DESY, Hamburg, Germany

3) Now at Centre de Physique des Particules, Faculté de Luminy, Marseille, France

4) On leave of absence form ITEP (Moscow)

5) Now at McGill University, Montreal, Canada

6) Now at Université de Montreal, Montreal, Canada

8) Inter-University Institute for Nuclear Science, Belgium

9) Now at CERN, Geneva, Switzerland

a) Supported by the German Bundesministerium für Forschung und Technologie under contract numbers 05-4HH22P and 05-4MU12P
} 


\section{Introduction}

Experiments at accelerators have already set very stringent limits on lepton flavour mixing angle, especially for $\nu_{\mu} \rightarrow \nu_{e}$ transitions [1-3].

The CHARM II experiment, designed to detect $\nu_{\mu} e$ and $\bar{\nu}_{\mu} e$ elastic scattering, is particularly suited for a measurement of the transition probability $P\left(\nu_{\mu} \rightarrow \nu_{e}\right)$ at high energy. The detector was exposed to the CERN-SPS wide band neutrino beam (average $\left.E_{\nu} \sim 20 \mathrm{GeV}\right)$ for five years and collected more than $10^{7} \nu_{\mu}\left(\bar{\nu}_{\mu}\right)$ charged current events. The apparatus allows clean identification of muons, good separation of hadronic from electromagnetic (e.m.) showers, and also some discrimination between showers initiated by a single electron and those induced by a $\pi^{0}$.

The method used in this search for oscillation consists in a comparison between the number of observed $\nu_{e}\left(\bar{\nu}_{e}\right)$ nucleon quasi-elastic interactions with those expected from the known contamination of $\nu_{e}\left(\bar{\nu}_{e}\right)$ in the beam. Any excess can be interpreted in terms of an oscillation probability, given - in a two neutrino scheme - by the well-known relation

$$
P=\sin ^{2} 2 \theta \sin ^{2} \frac{1.27 \Delta m^{2} L}{E}
$$

where $\theta$ is the lepton flavour mixing angle, $\Delta m^{2}$ is the difference of the square of the masses in $\mathrm{eV}^{2}, E$ is the neutrino energy in $\mathrm{GeV}, L$ is the mean distance between the neutrino source and the detector in $\mathrm{km}$ (in this experiment $L \sim 0.65 \mathrm{~km}$ ).

\section{Method of analysis}

We evaluated limits on $\nu_{\mu} \rightarrow \nu_{e}$ oscillation by comparing the observed number of quasi-elastic interactions, $\nu_{e} n \rightarrow e^{-} p$ and $\bar{\nu}_{e} p \rightarrow e^{+} n$, with the number of events expected from the $\nu_{e}\left(\bar{\nu}_{e}\right)$ contamination in the beam.

The observed number of events is obtained in the following way. The data collected are filtered in order to select events that appear in the detector as an isolated e.m. shower. From this sample we subtracted events generated by two background processes, namely $\nu_{\mu}$ scattering off electrons and single $\pi^{0}$ production from neutral current (NC) $\nu_{\mu} N$ scattering. While $\nu_{\mu}$ e scattering is easily removed by means of kinematical cuts, the $\pi^{0}$ background is evaluated making use of the difference in the signal released by an electron and a $\pi^{0}$ in a scintillation counter at the beginning of the shower development.

The expected number of events is computed in two steps. First the expected flux of $\nu_{e}\left(\bar{\nu}_{e}\right)$ is evaluated by Monte Carlo simulation of the production and decay of the parent pions and kaons. Then the cross-section for quasi-elastic scattering and the detection efficiency are taken into account. The latter is computed by analysing the quasi-elastic $\nu_{\mu} N \rightarrow \mu N^{\prime}$ events collected within the detector; the muon of the final state is replaced by an e.m. shower of the same energy and direction, and the full selection chain is applied to this sample.

\section{Data sample}

The data used in this analysis were collected in the period 1987-1991 by exposing the CHARM II detector to the CERN neutrino wide band beam for $\sim 2.6 \cdot 10^{19}$ protons on target. Depending on the polarity of the magnets focusing the charged neutrino parents, the main component of the beam was $\bar{\nu}_{\mu}$ or $\nu_{\mu}$. The integrated flux ratio was 1.2 .

The experimental apparatus, described in more detail in Ref. [4], consisted of a massive and low-density fine-grained calorimeter ( $\sim 700 \mathrm{t}$ weight, $\sim 35 \mathrm{~m} \mathrm{long})$ and of a 
muon spectrometer. The basic building element of the calorimeter was a $48 \mathrm{~mm}$ thick glass target corresponding to 0.5 radiation lengths, followed by a plane of plastic streamer tubes; the total number of calorimeter planes was 420 . Behind every group of five such modules a plane of $3 \mathrm{~cm}$ thick, $15 \mathrm{~cm}$ wide and $3 \mathrm{~m}$ long plastic scintillation counters was inserted to measure $d E / d x$. The low $Z$ material and the granularity were chosen to optimize the angular resolution for electron showers while the energy resolution achieved using a digital readout system of the streamer tubes was $\sigma(E) / E=0.09+0.15 / \sqrt{E(\mathrm{GeV})}$. The muon spectrometer consisted of magnetised iron toroids interspersed with scintillation counters and drift chambers. It measured the momentum of penetrating particles with a typical resolution of $\frac{\Delta p}{p}=13 \%$ at $20 \mathrm{GeV}$. Selected quasi-elastic $\nu_{\mu}\left(\bar{\nu}_{\mu}\right)$ and $\nu_{e}\left(\bar{\nu}_{e}\right)$ interactions appeared as a track reaching the spectrometer and as an e.m. shower in the calorimeter, respectively. While the spectrometer determined the charge of the muon, the calorimeter cannot separate electrons from positrons.

In order to obtain a clean sample for analysis, we selected events characterized by small hadronic activity near the vertex. They originate from the quasi-elastic interactions

$$
\nu_{l} n \rightarrow l^{-} p
$$

and

$$
\bar{\nu}_{l} p \rightarrow l^{+} n
$$

with $l=e, \mu$, and from resonance production and they have a clear signature in the CHARM II detector.

The starting sample for the selection of quasi-elastic $\nu_{e}$ candidates was obtained from the events satisfying the electron trigger [4] and the criteria for separation between e.m. and hadronic showers. The algorithms adopted for this separation (described in detail in Ref. [4]) were based on the pattern of hits in the streamer tubes and on the comparison between the total energy and that deposited close to the shower axis. Since the energy deposited in the scintillator played a crucial role in the analysis, only events whose vertex occured in a module that was followed by a scintillator plane were accepted. To avoid edge effects, the vertex itself was required to lie within a $145 \mathrm{~cm}$ radius around the detector axis, between plane 25 and 400 along the beam direction, and more than $1 \mathrm{~cm}$ inside the scintillator counters. Moreover, to further improve hadronic background rejection, the selected events were required to have a signal only in one of the scintillators of the following plane and only one hit in the first active tube layer of the shower.

Muon-neutrino quasi-elastic events were used to compute the expected number of $\nu_{e}$ quasi-elastic events, by replacing the muon with an electron shower. They were selected by a dedicated trigger which required a track in the spectrometer and subsequently in the offline analysis by discriminating against shower activity at the vertex. The rejection adopted corresponds to a hadronic energy threshold of $1.5 \mathrm{GeV}$. It was also required that the charge of muons be negative (positive) for the $\nu_{\mu}\left(\bar{\nu}_{\mu}\right)$ beams, respectively, and that the vertex lie between planes 300 and 400 of the calorimeter, sufficiently close to the muon spectrometer to ensure maximum geometrical acceptance. In addition, at most another track that did not go further than 30 planes in the calorimeter could be present in the event. This track could in fact be masked when the muon is replaced by an electron shower. The cuts on the radial coordinate of the vertex and on the multiplicity of hits in the vertex plane were also applied for the selection of the electron sample.

We note that the resulting efficiency for detecting quasi-elastic interactions is higher for $\bar{\nu}_{\mu}$ than for $\nu_{\mu}$ because of the recoiling proton in the latter case. The ratio of 'visible' cross-sections, a number which will be used later, is $\rho=1.49 \pm 0.05$. 


\section{Determination of observed and expected rates of $\nu_{e}$ events}

a) Observed number of events

The sample of e.m. showers described in the previous section, was analysed in order to separate the quasi-elastic $\nu_{e}$ events from the background. The data were divided in two intervals of shower energy: $5 \div 20 \mathrm{GeV}$ and $20 \div 35 \mathrm{GeV}$. The corresponding numbers of events are given in Table 1 under the heading 'e.m. showers'. We considered two energy bins because different energy spectra are expected for the three competing processes: $\nu_{e}$ quasi-elastic events coming from the $\nu_{e}$ contamination in the beam, $\nu_{e}$ quasi-elastic events coming from $\nu_{\mu}-\nu_{e}$ oscillation, and background from single $\pi^{0}$ production. In principle a higher sensitivity could be obtained in the low energy interval where the contamination of $\nu_{e}$ in the beam is lower. However, it turns out that the two regions give similar results and they are merged for the final result.

To determine the number of observed $\nu_{e}$ quasi-elastic events, the backgrounds from $\nu_{\mu}$ e scattering and single $\pi^{0}$ production were subtracted in the manner described below.

The elastic neutrino interactions on electrons were eliminated by a selection on the variable $E \theta^{2}$, where $E$ is the electron energy and $\theta$ is the angle of the recoiling electron. For kinematical reasons, $E \theta^{2}$ must be less than $2 m_{e} c^{2}$ for this type of events and therefore the outgoing electron is confined to small angles. Selecting leptons with $E \theta^{2} \geq 5 \mathrm{MeV}$ safely removed elastic scattering from the electron sample as well as inverse muon decay from the muon sample. In addition $E \theta^{2} \leq 72 \mathrm{MeV}$ was chosen to make use of efficiencies and knowledge of systematic errors studied in detail for the analysis that led to the determination of $\sin ^{2} \theta_{W}[5]$.

The other background process is single $\pi^{0}$ production in $\nu_{\mu}$ and $\bar{\nu}_{\mu}$ induced neutral current interactions. The conversion into $e^{-} e^{+}$pairs of photons produced in $\pi^{0}$ decay generates an e.m. shower which is very similar to that of a single electron, except at the very beginning. On a statistical basis, $e / \pi^{0}$ separation is possible by discriminating on the energy deposited near the origin of the shower. For the electron events selected with the criteria described above, this energy, $E_{\text {first }}$, is measured in the first scintillator that follows the vertex. It is roughly proportional to the number of charged particles crossing the scintillator, which would be odd in the case of an electron and even in the case of a converted photon. From an accurate simulation of events in the calorimeter [6], it was found that the most effective separation for our purpose is achieved by requiring $E_{\text {first }}<$ $8 \mathrm{MeV}$. This cut rejects $(89.8 \pm 4.4$ stat. \pm 6.5 syst. $) \%$ of the coherently and diffractively produced $\pi^{0}$, while accepting $(56.1 \pm 1.5$ stat. \pm 1.6 syst. $) \%$ of $\nu_{e}$ (or $\left.\bar{\nu}_{e}\right)$ quasi-elastic interactions in the neutrino beam and a slightly higher percentage in the antineutrino beam, $(58.2 \pm 1.4$ stat. \pm 2.3 syst. $) \%$.

The number of events, $N_{8}$, satisfying $E_{\text {first }}<8 \mathrm{MeV}$, is given in Table 1. Using these numbers, together with the total number of e.m. showers and with the efficiencies quoted above, it is possible to obtain the number of observed events, $N_{e}^{o b s}$, presented in Table 1. The error on $N_{e}^{o b s}$ is obtained by adding in quadrature statistical and systematic contributions. The distributions of $E_{\text {first }}$ for all the selected e.m. showers are shown in Fig.1, separately for different beam helicities and shower energy regions. Superimposed, in the same figure, are the $E_{\text {first }}$ distributions of simulated electron events, normalized to $N_{e}^{o b s}$. It can be seen that the $\pi^{0}$ background is larger for shower energies below $20 \mathrm{GeV}$. 
b) Expected number of events

Neutrinos of all flavours and helicities were present in the two beams with the exception of $\nu_{\tau}$, whose contribution was estimated to be less than $10^{-7}$ [7]. The contamination of $\nu_{e}$ and $\bar{\nu}_{e}$ was computed by a Monte Carlo simulation that started from a parametrization of the spectra of the secondary particles [8] produced by protons in the beryllium target. The data were required to reproduce the measured muon intensity at various radii and depths in the shield following the decay tunnel. The major uncertainty on the ratio $\nu_{e} / \nu_{\mu}$ originates from the lack of knowledge of the ratio of the neutrino parents, $K / \pi$. The total systematic uncertainty on the flux ratio was estimated to be $15 \%$ [9]. The calculations showed that the $\nu_{e}$ and $\bar{\nu}_{e}$ contamination in the beam (essentially coming from $K_{e 3}$ decay) was smaller at low energies. This motivated the choice of the two energy intervals.

The results of the simulation, relevant to this analysis, are summarized in Table 1. $R_{\phi}\left(R_{\bar{\phi}}\right)$ is the ratio of the fluxes of $\nu_{e}\left(\bar{\nu}_{e}\right)$ to the main neutrino component of the beam, $R_{w h}$ is the flux ratio of $\bar{\nu}_{\mu}$ over $\nu_{\mu}$ (or $\nu_{\mu}$ over $\bar{\nu}_{\mu}$ depending on the beam).

To compute the number of electron events from the beam contamination, we started from the observed number of $\nu_{\mu}$ quasi-elastic events corrected for inefficiencies of the trigger and of the spectrometer. In addition, we took into account the acceptance correction due to the different fiducial volume adopted for the analysis of e.m. showers and muon events (see Section 3 ). The overall multiplication coefficients have values in the range $4 \div 6$ and lead to the corrected numbers of events $N_{\mu}$ shown in Table 1.

Electron and muon neutrino (antineutrino) quasi-elastic events were not detected with equal efficiency. Their relative detection efficiency $\epsilon_{e / \mu}$ was obtained using an empirical approach to reduce systematic uncertainties due to nuclear effects. The method is based on the observation that at these energies the kinematics of the process is the same for $\nu_{e}$ and $\nu_{\mu}$ interactions. Starting from the selected muons, a weight was assigned to each event to account for the different neutrino spectra. The muon track was then replaced by a simulated electron shower of the same energy and direction as the muon. Each event was then required to go through the selection chain used for the hadronic background rejection and the relative detection efficiencies were computed in the two kinematical regions of interest, for both beams. The final values of $\epsilon_{e / \mu}$ are given in Table 1 . The last coefficient to be applied, $\epsilon_{\text {vertex }}$, is given in the next line of Table 1 . This factor takes into account the geometrical cuts on the vertex position (required to be in every fifth module and $1 \mathrm{~cm}$ away from the edge of the scintillation counters, see Section 3 ) and the requirement of only one scintillation counter hit in the first plane. A slight dependence from the kinematics of the events, hence small differences between the four values of $\epsilon_{\text {vertex }}$, is generated by the latter condition.

Both electrons and positrons contribute to the number of expected events, $N_{e}^{e x p}$ (Table 1). In the neutrino beam the total number of events (electrons plus positrons) is computed using the following expression separately for the two energy bins:

$$
N_{e}^{e x p}(\nu \text { beam })=\left[R_{\phi} \cdot \epsilon_{e / \mu}^{\nu} \cdot \epsilon_{v e r t e x}^{\nu}+R_{\bar{\phi}}^{\nu} \rho \cdot \epsilon_{e / \mu}^{\bar{\nu}} \cdot \epsilon_{v e r t e x}^{\bar{\nu}}\right] \cdot N_{\mu} .
$$

We recall that the factor $\rho$ (Section 3 ) takes into account the relative efficiency of detection of $\nu_{\bar{\mu}}$ compared to $\nu_{\mu}$ quasi-elastic interaction.

For the antineutrino beam, we have:

$$
N_{e}^{e x p}(\bar{\nu} \text { beam })=\left[R_{\phi} \cdot(1 / \rho) \cdot \epsilon_{e / \mu}^{\nu} \cdot \epsilon_{\text {vertex }}^{\nu}+R_{\bar{\phi}} \cdot \epsilon_{e / \mu}^{\bar{\nu}} \cdot \epsilon_{\text {vertex }}^{\bar{\nu}}\right] \cdot N_{\bar{\mu}} .
$$

It should be pointed out that our evaluation of $N_{e}^{e x p}$ could only underestimate the real number of expected $\nu_{e}$ interactions, since it does not take into account the events which were rejected in the $\nu_{\mu}\left(\bar{\nu}_{\mu}\right)$ quasi-elastic sample but which could be present in the selected 
electron sample. In fact, events with both a charged lepton and a $\pi^{0}$ in the final state and small hadronic activity at the vertex were considered as single electrons by the $E_{\text {first }}$ algorithm if the two e.m. showers overlapped and the photons from $\pi^{0}$ did not convert in the material between the decay point and the scintillator. This category of events is generated by $\nu_{e}\left(\bar{\nu}_{e}\right) \mathrm{CC}$ interaction of the type

$$
\nu_{e} n \rightarrow e^{-} N^{*} \quad \text { and } \quad \bar{\nu}_{e} p \rightarrow e^{+} N^{*}
$$

where $N^{*}$ is a baryonic resonance which might produce a single neutral pion in its decay. On the contrary, in the selected muon sample, they would have appeared as a track and an e.m. shower with the same origin and therefore they were mostly rejected by the requirements on the activity near the vertex. Since an accurate evaluation of this small contribution is difficult and our aim is to give an upper limit on the probability of oscillation, conservatively we neglected it.

\section{Results and discussions}

The results presented in Table 1 show no evidence for neutrino oscillation as the excess of observed over expected events $\left(N_{e}^{e x c}=N_{e}^{o b s}-N_{e}^{e x p}\right)$ is in all regions consistent with zero. Since electrons are not distinguished from positrons (i.e. $\nu_{e}$ interactions from $\bar{\nu}_{e}$ ), to convert $N_{e}^{e x c}$ into limits on neutrino oscillation one has to take into account the contribution coming from the wrong helicity neutrino contamination. The contribution depends on $R_{w h}$, the flux ratio of $\bar{\nu}_{\mu}$ over $\nu_{\mu}$ in the neutrino beam (and $\nu_{\mu}$ over $\bar{\nu}_{\mu}$ in the antineutrino beam), which is less than $10 \%$ for both beams and regions (Table 1 ). The probability of oscillation can be given separately for $\nu_{\mu}$ into $\nu_{e}$ and $\bar{\nu}_{\mu}$ into $\bar{\nu}_{e}$ simply by solving the following system of equations:

$$
\begin{aligned}
& N_{e}^{\text {exc }}(\nu \text { beam })=\left[P\left(\nu_{\mu} \rightarrow \nu_{e}\right) \cdot \epsilon_{e / \mu}^{\nu} \cdot \epsilon_{\text {vertex }}^{\nu}+R_{w h}^{\nu} \cdot \rho \cdot \epsilon_{e / \mu}^{\bar{\nu}} \cdot \epsilon_{\text {vertex }}^{\bar{\nu}} P\left(\bar{\nu}_{\mu} \rightarrow \bar{\nu}_{e}\right)\right] \cdot N_{\mu} \\
& N_{e}^{\text {exc }}(\bar{\nu} \text { beam })=\left[P\left(\bar{\nu}_{\mu} \rightarrow \bar{\nu}_{e}\right) \cdot \epsilon_{e / \mu}^{\bar{\nu}} \cdot \epsilon_{\text {vertex }}^{\bar{\nu}}+R_{w h}^{\bar{\nu}} \cdot(1 / \rho) \cdot \epsilon_{e / \mu}^{\nu} \cdot \epsilon_{\text {vertex }}^{\nu} P\left(\nu_{\mu} \rightarrow \nu_{e}\right)\right] \cdot N_{\bar{\mu}} .
\end{aligned}
$$

The $90 \%$ C.L. upper limits obtained by combining both energy regions are:

$$
\begin{aligned}
& P\left(\nu_{\mu} \rightarrow \nu_{e}\right)<4.7 \cdot 10^{-3} \\
& P\left(\bar{\nu}_{\mu} \rightarrow \bar{\nu}_{e}\right)<2.4 \cdot 10^{-3} .
\end{aligned}
$$

If $\mathrm{CP}$ invariance is valid, it is allowed to combine these results. A total excess of $265 \pm 178$ events is found and it corresponds to a limit of $2.8 \cdot 10^{-3}$ on $P\left(\nu_{\mu}, \bar{\nu}_{\mu} \rightarrow \nu_{e}, \bar{\nu}_{e}\right)$ at the $90 \%$ confidence level.

The curve delimiting the region in the parameters space is shown in Fig.2. For large mass differences $\left(\Delta m^{2}>200 e v^{2}\right), \sin ^{2} 2 \theta<5.6 \cdot 10^{-3}$ is obtained at the $90 \%$ C.L.; separately for neutrinos and antineutrinos the limits are $9.4 \cdot 10^{-3}$ and $4.8 \cdot 10^{-3}$, respectively. These results are comparable with the world best limits given by BNL-E776 [2] - $\sin ^{2} 2 \theta<3 \cdot 10^{-3}$ (for a mixture of neutrino and antineutrino beams) - and by BNL-E734 [1] $-\sin ^{2} 2 \theta<3.4 \cdot 10^{-3}$ (for a neutrino beam).

The search for oscillation was also performed using a different approach. This second analysis [10] relies on the observation that quasi-elastic interactions induced by $\nu_{e}$ 's coming from $\nu_{\mu}-\nu_{e}$ oscillation can be distinguished from other processes, leading to an e.m. shower in the final state, on the basis of the differences in the energy spectrum.

The data for this second analysis were those used for the study of $\nu_{\mu}$ e scattering [5]. The statistics is about 10 times larger in this latter case since scintillator planes were not used and, therefore, the cuts related to them were not applied. The energy spectrum of the e.m. showers was fitted with the contributions from diffractive and coherent $\pi^{0}$ production, 
and from quasi-elastic interactions owing to the $\nu_{e}$ contamination in the beam, with the energy dependence modeled in the way described in Ref. [5]. The contribution from $\nu_{\mu} e$ scattering was eliminated by the $E \theta^{2}$ cut described in Section 4 . In addition to these processes, a possible contribution of $\nu_{e}$ 's that originate from oscillation was considered. Their energy was distributed according to the Monte Carlo simulation that describes the beam. The contribution from oscillation was increased until the fit to the data became of poor quality and correspondingly upper limits on the oscillation parameters could be computed.

The results of this procedure turn out to be very similar to those obtained with the method of the first analysis. However, the computation of systematic errors affecting the result is more difficult. We consider this second approach as a confirmation of our results.

\section{Conclusions}

New limits on the probability of oscillation $\nu_{\mu} \rightarrow \nu_{e}$ and $\bar{\nu}_{\mu} \rightarrow \bar{\nu}_{e}$ have been set using quasi-elastic events collected in the CHARM II detector over a period of five years.

No significant excess of electron neutrino induced events has been found over what is expected from the beam composition. This allows us to state at the $90 \%$ C.L. that $\sin ^{2} 2 \theta$ is less than $9.4 \cdot 10^{-3}$ in the case of $\nu_{\mu}$ oscillation and less than $4.8 \cdot 10^{-3}$ in the case of $\bar{\nu}_{\mu}$, for $\Delta m^{2}>200 \mathrm{eV}^{2}$; combining neutrino and antineutrino data, the limit $\sin ^{2} 2 \theta<5.6 \cdot 10^{-3}$ is obtained.

\section{Acknowledgements}

We gratefully acknowledge the work of our technical collaborators who have contributed to the success of the experiment. The experiment has been made possible by grants from the Inter-University Institute for Nuclear Science (Belgium), CERN, the Bundesministerium für Forschung und Technologie (Germany), the Institute of Theoretical and Experimental Physics (Moscow, Russian Federation), the Istituto Nazionale di Fisica Nucleare(Italy), and the Turkish Scientific and Research Council, TÜBİTAK (Turkey); we gratefully acknowledge their support. We thank the staff of the CERN Super Proton Synchrotron and the neutrino beam crew for their competent assistance ensuring the excellent performance of their facility.

\section{References}

[1] L.A. Ahrens et al., Phys. Rev. D31 (1985) 2732.

[2] L. Borodovsky et al., Phys. Rev. Lett. 68 (1992) 274.

[3] CHARM Collab., F. Bergsma et al., Z. Phys. C 40 (1988) 171.

[4] CHARM II Collab., K. De Winter et al., Nucl. Instr. Meth. A 278 (1989) 670.

CHARM II Collab., D. Geiregat et al., Nucl. Instr. Meth. A 325 (1993) 92.

[5] CHARM II Collab., D. Geiregat et al., Phys. Lett. B 259 (1991) 499.

CHARM II Collab., P. Vilain et al., CERN-PPE/94-124.

[6] T. Voss, Ph.D. thesis, Hamburg University, 1993 (unpublished).

[7] CHORUS Collab., M. de Jong et al., CERN-PPE/93-131.

[8] H.W. Atherton et al., CERN Yellow Report 80-07 (1980).

[9] CHARM II Collab., P. Vilain et al., Phys. Lett. B 281 (1992) 159.

[10] M. Serin-Zeyrek, Ph.D. thesis, M.E.T.U., Ankara, 1992 (unpublished).

[11] C. Angelini et al., Phys. Lett. B 179 (1986) 307. 
Fig. 1 Distribution of the energy deposited in the first scintillator after the vertex, $E_{\text {first }}$, for selected e.m. shower candidates in the shower energy ranges $5 \div 20 \mathrm{GeV}$ and $20 \div 35 \mathrm{GeV}$. The black histograms are the Monte Carlo generated distributions for electrons normalized to the number of $\nu_{e}\left(\bar{\nu}_{e}\right)$ quasi-elastic interactions observed in each sample.

Fig. 2 The region on the right of the full line is excluded at the $90 \%$ C.L. in the $\sin ^{2} 2 \theta-$ $\Delta m^{2}$ plane by this analysis. The limits obtained by other neutrino accelerator experiments (BNL-E734 [1], BNL-E776 [2], CHARM [3], BEBC [11]) are also shown.

\section{Table caption}

Table 1 Analysis results in the low $(5 \div 20 \mathrm{GeV})$ and high $(20 \div 35 \mathrm{GeV})$ energy regions for neutrino and antineutrino beams. 
Table 1

\begin{tabular}{|c|c|c|c|c|}
\hline Beam & \multicolumn{2}{|c|}{$\nu$} & \multicolumn{2}{|c|}{$\bar{\nu}$} \\
\hline Shower energy & $5 \div 20 \mathrm{GeV}$ & $20 \div 35 \mathrm{GeV}$ & $5 \div 20 \mathrm{GeV}$ & $20 \div 35 \mathrm{GeV}$ \\
\hline e.m. showers & 2641 & 673 & 3208 & 766 \\
\hline$N_{8}$ & 385 & 141 & 520 & 211 \\
\hline$N_{e}^{o b s}$ & $252 \pm 59$ & $158 \pm 27$ & $402 \pm 68$ & $277 \pm 33$ \\
\hline$R_{\phi}\left(\cdot 10^{-3}\right)$ & $2.6 \pm 0.4$ & $6.3 \pm 0.9$ & $1.9 \pm 0.3$ & $3.6 \pm 0.5$ \\
\hline$R_{\bar{\phi}}\left(\cdot 10^{-3}\right)$ & $0.9 \pm 0.1$ & $1.3 \pm 0.2$ & $2.5 \pm 0.4$ & $6.5 \pm 1.0$ \\
\hline$R_{w h}(\%)$ & 5.8 & 4.3 & 9.1 & 9.6 \\
\hline$N_{\mu}$ & 494,649 & 234,169 & 935,418 & 385,998 \\
\hline$\epsilon_{e / \mu}(\%)$ & $59 \pm 6$ & $52 \pm 5$ & $65 \pm 6$ & $62 \pm 6$ \\
\hline$\epsilon_{\text {vertex }}(\%)$ & $12.6 \pm 0.2$ & $11.6 \pm 0.4$ & $13.5 \pm 0.2$ & $12.4 \pm 0.4$ \\
\hline$N_{e}^{e x p}$ & $149 \pm 27$ & $122 \pm 22$ & $300 \pm 53$ & $253 \pm 46$ \\
\hline$N_{e}^{e x c}$ & $103 \pm 65$ & $36 \pm 34$ & $102 \pm 86$ & $24 \pm 56$ \\
\hline
\end{tabular}



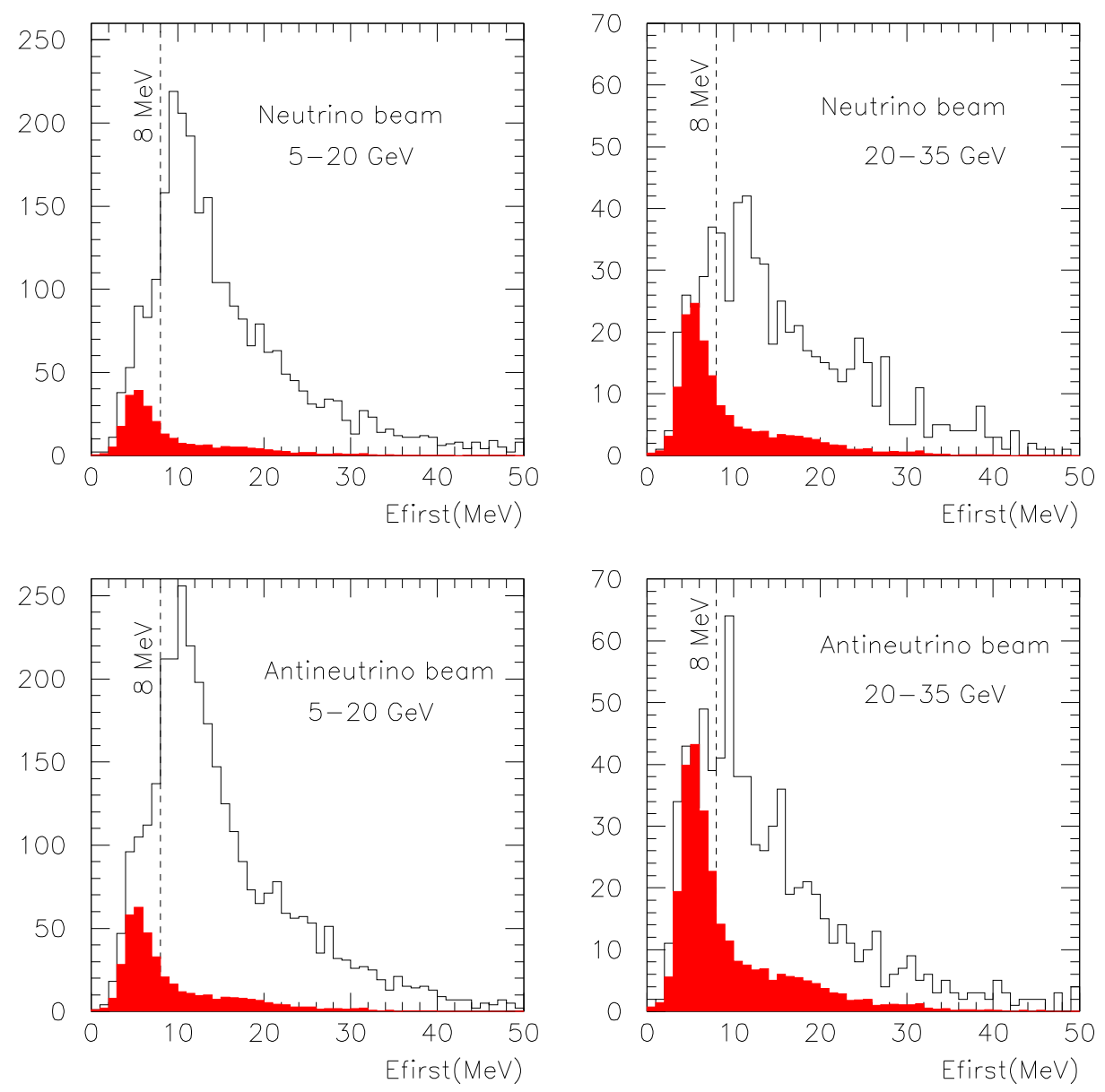

Fig. 1 


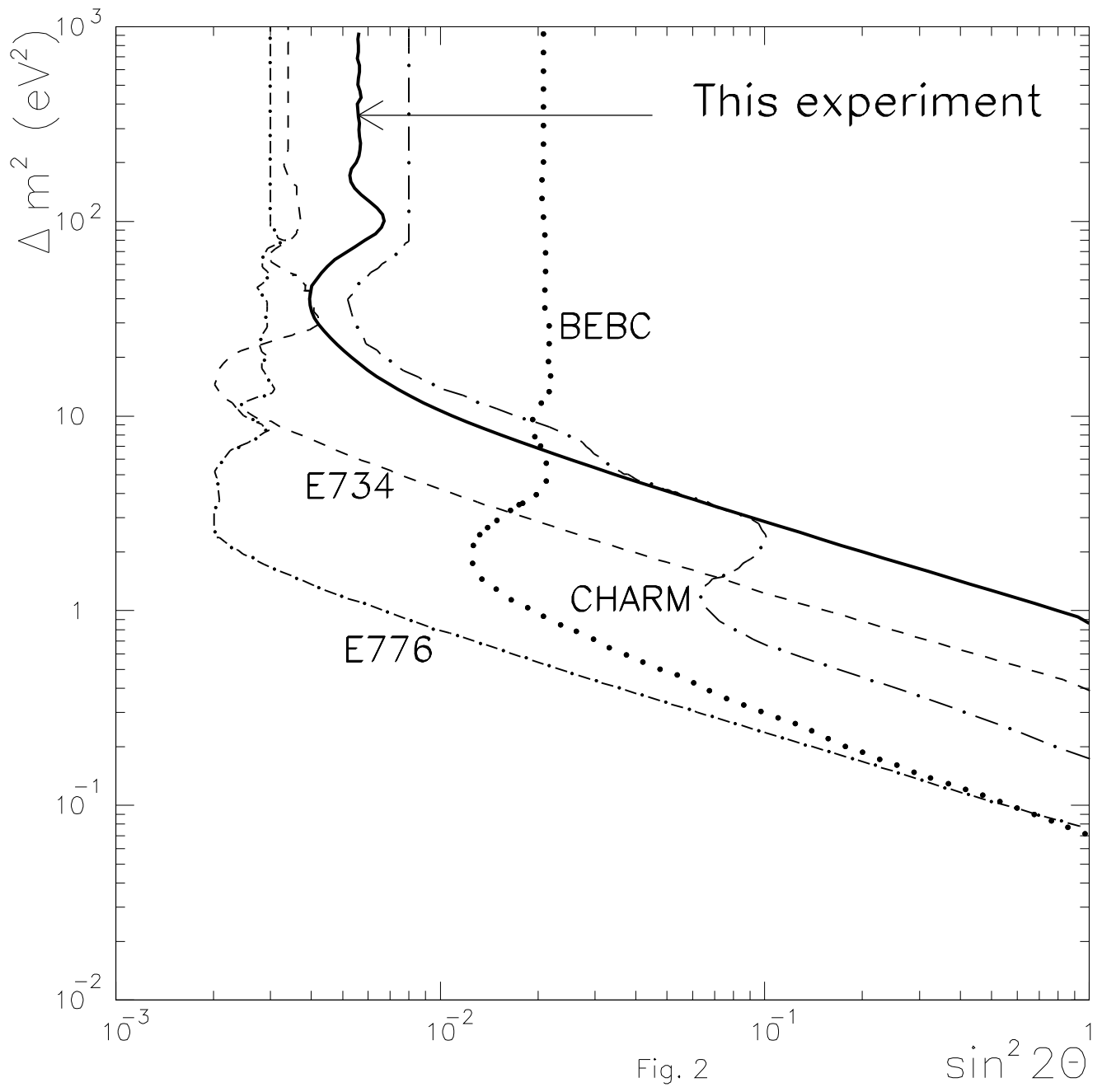

